DOI: 10.12957/demetra.2018.34088

\title{
Insegurança alimentar e nutricional e fatores de risco para doenças crônicas não transmissíveis em catadores de resíduos sólidos
}

\section{Food and nutritional insecurity and risk factors for chronic noncommunicable diseases among solid waste collectors}

Maria Rafaela Martins de Oliveira'

Roseane Saraiva de Santiago Lima²

Francisco Regis da Silva ${ }^{3}$

Luisa Maria Oliveira Pinto ${ }^{4}$

Rafaella Maria Monteiro Sampaio ${ }^{5}$

1 Universidade Federal do Ceará, Hospital Universitário Walter Cantídio, Programa de Residência Integrada Multiprofissional em Atenção Hospitalar à Saúde. Fortaleza, CE, Brasil.

2 Instituto Federal de Educação, Ciência e Tecnologia do Ceará, Curso de Nutrição. Limoeiro do Norte, CE, Brasil.

${ }^{3}$ Universidade Estadual do Ceará, Programa de Pós-Graduação em Saúde Coletiva. Fortaleza, CE, Brasil.

${ }^{4}$ Hospital Geral de Fortaleza, Ambulatório de Cardiologia. Fortaleza, CE, Brasil.

${ }^{5}$ Universidade de Fortaleza, Centro Universitário Estácio do Ceará, Curso de Nutrição. Fortaleza, CE, Brasil.

Correspondência / Correspondence

Francisco Regis da Silva

E-mail: regisfrs@hotmail.com

\section{Resumo}

Estudos têm demonstrado uma relação entre a situação de insegurança alimentar e nutricional com doenças crônicas não transmissíveis (DCNT), destacando que carências nutricionais e fome não são as únicas formas de expressão dessa condição. Desta forma, objetivou-se avaliar a ocorrência de insegurança alimentar e nutricional e os fatores de risco para desenvolvimento de DCNT em catadores de resíduos sólidos em um município do interior cearense. Realizou-se estudo transversal com 32 trabalhadores, com idades de 20 a 66 anos. Aplicou-se um questionário estruturado contendo dados de identificação, socioeconômicos, dietéticos e antropométricos. A insegurança alimentar e nutricional foi avaliada por meio da Escala Brasileira de Insegurança Alimentar (EBIA). Observou-se insegurança alimentar e nutricional em $100 \%$ da amostra, baixa escolaridade $(78,12 \%)$ e baixa renda $(87,5 \%)$, prevalência de sobrepeso $(25,0 \%)$ e obesidade $(31,26 \%)$ e insuficiente consumo de frutas, verduras e hortaliças. A proporção de insegurança alimentar moderada e grave foi alta, e os trabalhadores apresentaram fatores de risco para o desencadeamento de DCNT.

Palavras-chave: Catadores. Estado Nutricional. Doenças Crônicas. Nutrição em Saúde Pública. 


\section{Abstract}

Studies have shown relationship between food and nutritional insecurity with chronic noncommunicable diseases (CND), noting that nutritional deficiencies and hunger are not the only forms of expression of this condition. In This study aimed to evaluate the occurrence of food and nutritional insecurity and the risk factors for the development of CND's among solid waste collectors in a municipality in the interior of Ceara, Brazil. A cross-sectional study was carried out with 32 workers, aged 2066 years. A structured questionnaire containing identification, socioeconomic, dietary and anthropometric data was applied. Food and nutritional insecurity was evaluated through the Brazilian Scale of Food Insecurity (EBIA). There was food and nutritional insecurity in $100 \%$ of the sample, low schooling $(78.12 \%)$ and low income $(87.5 \%)$, prevalence of overweight (25.0\%) and obesity (31.26\%) and insufficient consumption of fruits, vegetables and vegetables. The proportion of food insecure moderate and severe was high, and workers presented risk factors for the onset of CND.

Keywords: Collectors. Nutritional status. Chronic diseases. Nutrition in Public Health.

\section{Introdução}

O conceito de segurança alimentar e nutricional (SAN) surgiu no período da Primeira Guerra Mundial e vem sendo amplamente discutido nos dias de hoje. Inicialmente, acreditava-se que o problema estava relacionado à escassez de alimentos, mas o aumento da produção desses não revogou os problemas de fome e desnutrição. Passou-se, então, a verificar que o problema de insegurança alimentar não estava relacionado com a carência, mas com a falta (pouca e/ou deficiente) de acesso em decorrência da pobreza. ${ }^{1}$

Neste sentido, entende-se por SAN o direito de todos ao acesso regular e permanente a alimentos de qualidade, em quantidades suficientes, sem comprometer as demais necessidades essenciais, tendo como base uma alimentação promotora de saúde, que respeite a diversidade cultural e que seja ambiental e socialmente sustentáveis. ${ }^{2-4}$

Desse modo, a SAN vem ocupando de forma crescente a agenda pública. Um importante passo foi o reconhecimento da alimentação como um direito humano, introduzido na Emenda 
Constitucional 64, em fevereiro de 2010. A Lei federal $n^{\circ}$ 11.346, de 15 de setembro de 2006 , conhecida como Lei Orgânica de Segurança Alimentar e Nutricional (LOSAN), criou o Sistema Nacional de Segurança Alimentar e Nutricional (SISAN), que tem por objetivo proporcionar a garantia desse direito por meio de políticas públicas, planos e ações, bem como promover o acompanhamento e o monitoramento desses. ${ }^{5,6}$

Apesar disso, estima-se que 1,02 bilhão de pessoas, ou seja, 14,3\% da população mundial, não tenham acesso a alimentos em quantidades satisfatórias para o suprimento de suas necessidades nutricionais básicas, ou se encontrem em situação de fome contínua, configurando uma situação de insegurança alimentar e nutricional grave. ${ }^{5}$

Estudos têm demonstrado relação entre a situação de insegurança alimentar com sobrepeso e obesidade, bem como com as doenças crônicas não transmissíveis (DCNT). Ressalta-se, assim, que carências nutricionais e fome não são as únicas formas de expressão dessa condição. ${ }^{7,8}$

No Brasil, assim como em outros países do mundo, o padrão alimentar atual inclui um consumo insuficiente de frutas, verduras e legumes. A carência da ingestão desses grupos alimentares está entre os principais fatores de risco para a carga global de doenças no mundo, incluindo câncer e demais DCNTs, como obesidade, diabetes tipo 2 e doenças cardiovasculares. ${ }^{9,10}$

Apesar de o tema "fatores de risco para DCNT" ser bastante abordado pela literatura, com referências inclusive para sexo, idade, escolaridade e nível de renda, pouco se sabe sobre as particularidades da distribuição destes fatores entre as diferentes profissões, sobretudo entre catadores de resíduos sólidos. É preciso considerar que, além de seu ambiente de trabalho ser propício ao desenvolvimento de doenças de cunho infeccioso, esses trabalhadores também, por seu estado de insegurança alimentar e nutricional, corroboram para a suscetibilidade a DCNTs. Portanto, estão inseridos em um contexto que induz ao aumento progressivo destas doenças, que poderão ser potencializadas pela condição de pobreza e desamparo em que vivem. ${ }^{11}$

Faz-se necessário, assim, analisar os riscos nutricionais em que se encontram os catadores de resíduos sólidos, visto que esses estão inseridos de forma precária e desigual no atual sistema econômico. Essa população busca na coleta de resíduos sólidos uma alternativa de sobrevivência, ficando exposta, de forma direta e constante, a diversos fatores de risco à saúde.

Os estudos relacionados à alimentação e às condições de saúde nessa população ainda são bastante restritos; por esse motivo, esta pesquisa teve como objetivo avaliar a ocorrência de insegurança alimentar e nutricional e os fatores de risco para desenvolvimento de DCNTs em catadores de resíduos sólidos em um município do interior cearense. 


\section{Metodologia}

Foi desenvolvido um estudo do tipo quantitativo de caráter transversal e descritivo, realizado com os catadores de resíduos sólidos do município de Russas, Ceará, durante o mês de fevereiro de 2015.

Foram incluídos no estudo os catadores de rua e de lixão que fazem parte da Associação dos Catadores de Materiais Recicláveis de Russas (ASCAMARRU). O cadastro dos associados foi solicitado à entidade de promoção e atuação social Caritas Diocesana.

A população era composta por 55 trabalhadores e o tamanho amostral calculado foi de 32 indivíduos, com nível de confiança de 95\%, segundo cálculo para determinação da amostra com base na estimativa da média populacional proposta por Triola. ${ }^{12}$

A coleta dos dados foi realizada no galpão de reciclagem da ASCAMARRU nos dias de reuniões e cursos promovidos pela Caritas Diocesana. Tais dados foram levantados por meio de um questionário estruturado, com os seguintes tópicos: dados pessoais, grau de escolaridade, renda per capita, condição de insegurança alimentar e nutricional, dados antropométricos e consumo de frutas, verduras e legumes.

A escolaridade foi avaliada de acordo com a padronização realizada pelo Inquérito Nacional para Fatores de Risco de DCNT (VIGITEL), que define como baixa escolaridade oito anos ou menos de estudo. ${ }^{13}$ A renda foi avaliada considerando como linha de pobreza um valor per capita inferior a meio salário mínimo por mês, como adotado pelo Programa das Nações Unidas para o Desenvolvimento (PNUD). ${ }^{14}$

O diagnóstico da insegurança alimentar foi realizado por meio da Escala Brasileira de Insegurança Alimentar (EBIA), composta por 15 perguntas fechadas com respostas positivas ou negativas, relativa à percepção dos entrevistados sobre a situação alimentar vivida pela família nos últimos três meses anteriores à entrevista. Nas respostas positivas, atribui-se o valor "1" (um), e para as respostas negativas, o valor "0" (zero). O somatório dos pontos possibilitou classificar a situação alimentar em quatro categorias: 1) Segurança alimentar, quando não há problemas de acesso aos alimentos, em termos qualitativos e quantitativos, e não há preocupação de que os alimentos venham a faltar no futuro; 2) Insegurança alimentar leve (IAL), quando há preocupação com a falta de alimentos no futuro próximo, retratando um componente psicológico de insegurança; 3) Insegurança alimentar moderada (IAM), quando há situação de comprometimento da qualidade da alimentação na busca por manter a quantidade necessária; 4) Insegurança alimentar grave (IAG), condição em que há deficiência quantitativa de alimento, levando à situação de fome. ${ }^{15,16}$

Para a avaliação do estado nutricional, aferiram-se peso e estatura, e posteriormente calculouse o índice de massa corporal (IMC), o qual se refere ao peso (Kg) dividido pela estatura ao quadrado $\left(\mathrm{m}^{2}\right)$. Utilizou-se como referência a classificação proposta pela Organização Mundial da Saúde (OMS). ${ }^{17}$ 
A circunferência da cintura (CC) foi aferida para identificar a gordura abdominal e os riscos para o desenvolvimento de doenças cardiovasculares. A medida da CC foi realizada na menor curvatura localizada entre as costelas e a crista ilíaca; quando não era possível identificar a menor curvatura, a leitura era feita dois centímetros acima da cicatriz umbilical. Os pontos de corte adotados foram os preconizados pela OMS, ${ }^{17}$ que determina como risco valores iguais ou maior que $80 \mathrm{~cm}$ para mulheres e $94 \mathrm{~cm}$ para homens.

O consumo de frutas, verduras e legumes foi quantificado de acordo com Mendes \& Catão, ${ }^{18}$ a partir das seguintes perguntas: "Com que frequência você consume frutas ou suco de frutas naturais?" e "Com que frequência você consume verduras e legumes?". Os trabalhadores foram orientados a optar por uma das cinco alternativas para o consumo: diário, semanal, mensal, raramente ou nunca. O participante deveria responder sobre o número de vezes por dia, semana ou mês em que consumia o citado alimento, e em seguida, quantas porções do mesmo eram habitualmente consumidas por vez.

Para facilitar o entendimento, os participantes da pesquisa eram informados sobre o conceito de "porção", sendo dados alguns exemplos de porção de frutas e verduras, com base no Guia Alimentar para a População Brasileira. Foi considerado positivo o consumo mínimo diário de 400 g ou cinco porções de 80 g cada. ${ }^{19}$

O banco de dados foi organizado no Microsoft Office Excel ${ }^{\circledR}$, 2010. A análise estatística constou de descrição das frequências das variáveis, percentual, média e desvio padrão, utilizando o Software Epi info versão 6.04 .

A participação dos trabalhadores no estudo foi condicionada à assinatura de um Termo de Consentimento Livre e Esclarecido (TCLE). O projeto de pesquisa foi aprovado pelo Comitê de Ética em Pesquisas (CEP) do Instituto Federal de Educação Ciências e Tecnologia do Ceará (IFCE), com parecer $n^{\circ} 1.176 .770$.

\section{Resultados}

A amostra foi composta por 32 trabalhadores, a maioria, 65,62\% $(n=21)$ do gênero feminino. A idade dos entrevistados variou entre 20 e 63 anos, sendo a média de idade das mulheres $(35,8$ $\pm 3,13$ ) menor que a dos homens $(44,27 \pm 3,52)$. O tempo médio de trabalho no "lixão" foi de 6,14 anos (DP $\pm 1,47$ ), com o mínimo de três meses e o máximo de 36 anos, podendo-se observar maior prevalência de tempo das mulheres, como demonstrado na tabela 1 .

Entre os participantes da pesquisa, 100\% apresentaram insegurança alimentar: para 12,50\% desses indivíduos, essa insegurança alimentar foi considerada leve; para 34,37\%, moderada; e para $53,13 \%$, grave (tabela 2 ). 


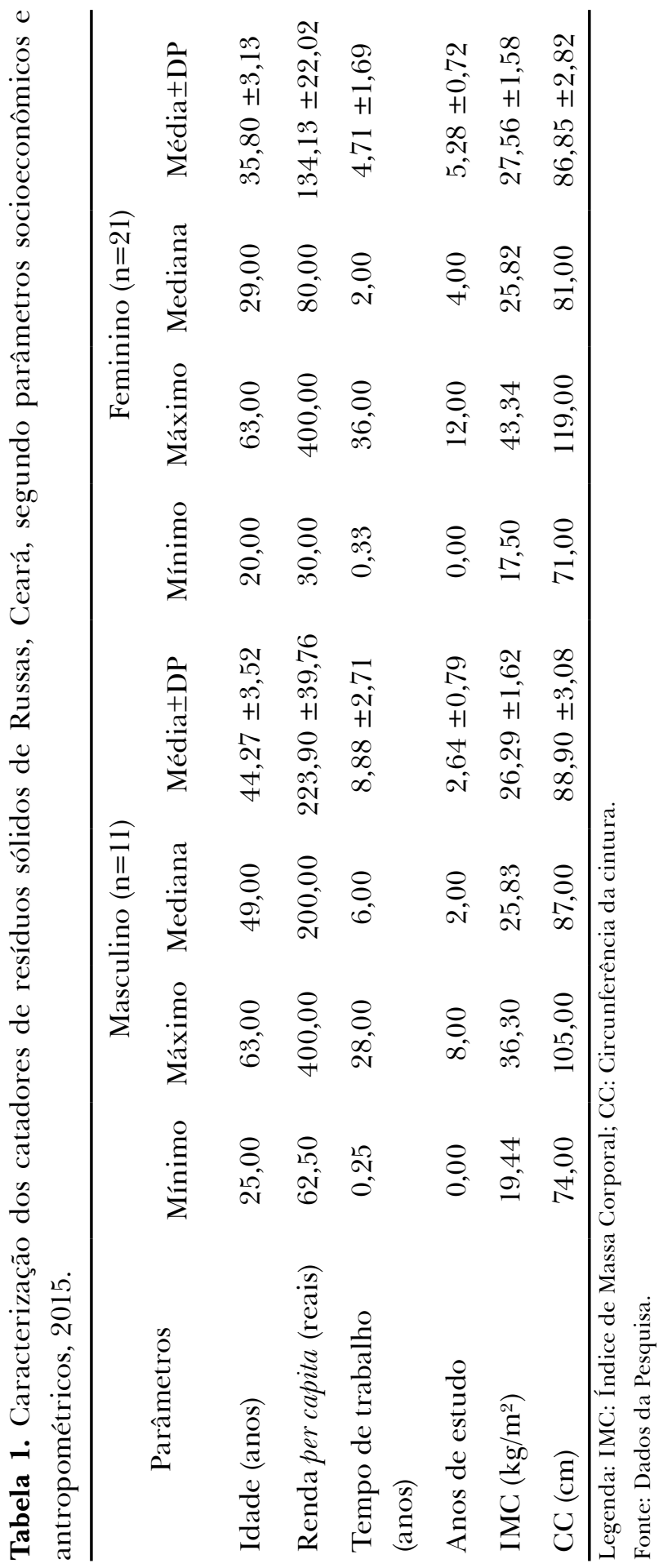


Tabela 2. Prevalências de insegurança alimentar de acordo com as características socioeconômicas, antropométricas e dietéticas em catadores de resíduos sólidos de Russas, Ceará, 2015.

\begin{tabular}{|c|c|c|c|c|}
\hline Variáveis & $\begin{array}{c}\text { IAL } \\
\%\end{array}$ & $\begin{array}{c}\text { IAM } \\
\%\end{array}$ & $\begin{array}{c}\text { IAG } \\
\%\end{array}$ & $\begin{array}{c}\text { Total } \\
\%\end{array}$ \\
\hline \multicolumn{5}{|l|}{ Insegurança Alimentar } \\
\hline & 12,50 & 34,37 & 53,13 & 100,00 \\
\hline \multicolumn{5}{|l|}{ Renda per capita } \\
\hline < 1/2 Salário mínimo & 12,50 & 31,25 & 43,75 & 87,50 \\
\hline$\geq 1 / 2$ Salário mínimo & 0,00 & 3,12 & 9,38 & 12,50 \\
\hline \multicolumn{5}{|l|}{ Anos de estudo } \\
\hline$<8$ anos de estudo & 9,38 & 25,00 & 43,74 & 78,12 \\
\hline$\geq 8$ anos de estudo & 3,12 & 9,38 & 9,38 & 21,88 \\
\hline \multicolumn{5}{|l|}{$I M C$} \\
\hline Desnutrição & 0,00 & 6,25 & 3,12 & 9,37 \\
\hline Eutrofia & 9,38 & 3,12 & 21,87 & 34,37 \\
\hline Sobrepeso & 3,12 & 12,50 & 9,38 & 25,00 \\
\hline Obesidade & 0,00 & 12,50 & 18,76 & 31,26 \\
\hline \multicolumn{5}{|l|}{$C C$} \\
\hline Sem risco & 9,38 & 15,63 & 24,99 & 50,00 \\
\hline Risco & 3,12 & 18,76 & 28,12 & 50,00 \\
\hline \multicolumn{5}{|l|}{ Consumo de Frutas } \\
\hline Diariamente & 3,12 & 0,00 & 3,12 & 6,24 \\
\hline Semanalmente & 0,00 & 15,63 & 3,12 & 18,75 \\
\hline Mensalmente & 3,12 & 3,12 & 6,25 & 12,49 \\
\hline Raramente/Nunca & 6,25 & 15,63 & 40,64 & 62,52 \\
\hline \multicolumn{5}{|l|}{ Consumo de verduras e Legumes } \\
\hline Diariamente & 3,12 & 3,12 & 9,38 & 15,62 \\
\hline Semanalmente & 3,12 & 6,25 & 3,12 & 12,49 \\
\hline Mensalmente & 0,00 & 0,00 & 0,00 & 0,00 \\
\hline Raramente/Nunca & 6,25 & 25,00 & 40,64 & 71,89 \\
\hline
\end{tabular}

Legenda: IAL: Insegurança alimentar leve; IAM: Insegurança Alimentar Moderada; IAG: Insegurança Alimentar Grave; IMC Índice de Massa Corporal; CC: Circunferência da cintura.

Fonte: Dados da Pesquisa. 
Embora 62,50\% dos entrevistados participassem de algum programa de auxílio governamental, 87,50\% possuíam renda per capita inferior a meio salário mínimo, situação que foi vista em todos os níveis de insegurança alimentar, sendo mais crítica de acordo com a gravidade dessa insegurança.

Em relação ao tempo de estudo, notou-se que 78,12\% estudaram menos de oito anos e assim como a renda per capita, a quantidade de entrevistados com baixa escolaridade foi maior conforme se elevava o grau da insegurança alimentar.

Quanto ao estado nutricional, percebeu-se que apenas $34,37 \%$ dos entrevistados apresentaram eutrofia segundo o IMC, e que 56,26\% estavam entre as classificações de sobrepeso e obesidade. O maior percentual de obesos foi observado entre os trabalhadores com insegurança alimentar grave.

A circunferência da cintura esteve inadequada em $50 \%$ dos participantes, principalmente para aqueles com maior grau de insegurança alimentar.

Percebeu-se deficiência no consumo de frutas, verduras e legumes, uma vez que 62,50\% dos entrevistados relataram ausência do consumo de frutas e $71,88 \%$ disseram nunca comer verduras e legumes. Assim como os demais indicadores de doenças crônicas, a quantidade de pessoas que não consumiam esses alimentos foi maior de acordo com a intensidade da insegurança alimentar.

\section{Discussão}

A insegurança alimentar é um fenômeno que vem sendo observado em todo o mundo, tanto nos países em desenvolvimento quanto nos desenvolvidos, comprometendo assim a saúde e o bem-estar de 963 milhões de pessoas. ${ }^{18}$

No Brasil, a Pesquisa Nacional por Amostragem de Domicílios (PNAD), ${ }^{20}$ realizada em 2013, identificou insegurança alimentar em 25,8\% dos moradores de domicílios particulares, e a prevalência de insegurança alimentar grave foi de 3,6\%. As condições mais precárias foram observadas na Região Nordeste, onde a prevalência global de insegurança alimentar era de 45,0\%, e a de insegurança alimentar grave, $5,6 \%$.

A prevalência geral de insegurança alimentar entre os catadores de resíduos sólidos de RussasCE foi muito superior à observada na Região Nordeste. A insegurança alimentar grave desses trabalhadores $(53,13 \%)$ foi maior que o somatório de todos os graus de insegurança na referida região. Porém, deve-se considerar que o presente artigo trabalhou com uma amostra especifica, constituída de uma população com precárias condições de subsistência.

O Instituto Brasileiro de Análises Sociais e Econômicas (IBASE) ${ }^{21}$ avaliou, em 2008, a condição de segurança alimentar das famílias beneficiadas pelo Programa Bolsa Família (PBF) nas diferentes regiões do país. Os favorecidos pelo PBF constituem, em si, um grupo de menor renda e sob maior vulnerabilidade a privações alimentares, assim como os catadores de resíduos sólidos, o 
que possibilita a comparação entre os dois grupos. Observou-se que, no Nordeste, $87,1 \%$ dos que recebiam o auxílio governamental encontravam-se em situação de insegurança alimentar, valor inferior ao observado no presente estudo. A prevalência de insegurança alimentar leve $(22,2 \%)$ e moderada $(38,7 \%)$ foi maior entre os beneficiados do PBF, enquanto a insegurança alimentar grave foi duas vezes maior nos catadores.

Santos et al. ${ }^{22}$ avaliaram a insegurança alimentar em catadores de resíduos sólidos de BrasíliaDF, utilizando uma escala curta de insegurança alimentar validada no Brasil, e observaram a prevalência de 75,0\% das famílias expostas a tal situação, número também inferior ao encontrado nesta pesquisa.

Alguns estudos têm relatado associação entre insegurança alimentar e doenças crônicas, tais como diabetes, obesidade e hipertensão. Terrell et al. ${ }^{23}$ pesquisaram a associação entre insegurança alimentar e os riscos de desenvolvimento de DCNTs, e observaram que os indivíduos em situação de insegurança alimentar estavam mais propensos ao aparecimento de diabetes. Além disso, o estudo apresentou como resultado um maior descontrole da glicemia, pressão arterial e proteinúria em indivíduos portadores de diabetes, hipertensão e doença renal crônica, respectivamente, que se encontravam em insegurança alimentar, quando comparados àqueles com segurança alimentar.

A condição de insegurança alimentar, por si só, favorece o surgimento de DCNTs, por meio da redução e da privação de nutrientes indispensáveis à saúde. Além disso, existem diversos outros fatores associados a esta condição que também predispõem a tais doenças. O estudo em questão analisou, dentre esses fatores, renda, escolaridade, estado nutricional e consumo de frutas e verduras.

As DCNTs atingem indivíduos de todas as classes socioeconômicas e, de forma mais intensa, aqueles pertencentes a grupos vulneráveis com baixa escolaridade e renda. ${ }^{24}$ Anschau et al., ${ }^{16}$ ao estudarem os beneficiados de programas de transferência de renda, residentes no município de Toledo-PR, notaram que quanto maior a escolaridade, mais distante o domicílio está da condição de insegurança alimentar. Em relação à renda, Anschau et al. ${ }^{16}$ também encontraram forte associação, mostrando que quanto menor a renda per capita, maiores as chances de a família apresentar insegurança alimentar moderada e grave, condições semelhantes às encontradas entre os catadores deste estudo.

O excesso de peso, incluindo as condições de sobrepeso e obesidade, constitui o sexto fator mais importante para a carga global de doenças, em razão de sua associação com várias DCNTs, incluindo doenças cardiovasculares, como hipertensão arterial e acidente vascular cerebral, diabetes, câncer de colo, reto, mama, entre outras. No Brasil, a prevalência de excesso de peso na população adulta aumentou de 43,2\% em 2006 para 51,0\% em 2012. O somatório dos níveis de sobrepeso e obesidade deste estudo foi semelhante aos encontrados por Freitas \& Antunes, ${ }^{25}$ realizado com operários de uma usina de reciclagem de Maracanaú, Ceará. Nesta, o percentual 
de pessoas com excesso de peso foi de $57,0 \%$, mas quando se analisou a obesidade de forma isolada, o presente estudo apresentou quase o dobro do encontrado pelos referidos autores (18,7\%). Bittencourt \& Muttoni ${ }^{26}$ avaliaram o estado nutricional de trabalhadores de cooperativas de reciclagem de Porto Alegre-RS e encontraram um percentual de indivíduos sobrepesados e obesos $(66,7 \%)$ superior aos estudos supracitados, e um total de obesos $(27,8 \%)$ maior que o encontrado em Maracanaú-CE, mas ainda inferior à dos catadores deste estudo.

Em se tratando de IMC e SAN, os dados deste artigo corroboram os achados de Santos et al., ${ }^{4}$ em que o déficit nutricional é pouco proveniente e as prevalências de excesso de peso e obesidade são bastante elevadas. Velásquez-Melendez et al. ${ }^{27}$ encontraram associação entre obesidade apenas para insegurança alimentar moderada, dado que contradiz o presente estudo, que encontrou grande frequência de obesidade também no grupo com insegurança alimentar grave.

Apesar de o IMC ser o indicador mais utilizado na prática clínica, ele possui limitações para diferenciar o peso atribuído ao músculo ou à gordura. Desta forma, recomenda-se a utilização do mesmo associado a outros métodos de composição corpórea. ${ }^{28}$

O indicador de circunferência da cintura está associado ao risco para o aparecimento de doenças cardiovasculares. No presente estudo, o risco estava acima do limite indicado para 50,0\% dos participantes, valor inferior ao demonstrado por Bittencourt e Muttoni ${ }^{26}(74,0 \%)$ em estudo com trabalhadores de cooperativas de reciclagem de Porto Alegre-RS.

Pesquisas mostram que as alterações na dieta têm efeitos positivos e negativos na saúde. Uma alimentação inadequada, rica em gorduras, com alimentos altamente refinados e processados, pobre em frutas, verduras e legumes, está associada ao aparecimento de diversas DCNTs, inclusive câncer. Oliveira et al.," ${ }^{11}$ em estudo com trabalhadores da usina de triagem de materiais recicláveis do município de Viçosa-MG, identificaram consumo insuficiente de fibras nessa população, em que $52,0 \%$ e 89,0\% dos participantes relataram não possuir hábito de consumir hortaliças e verduras, respectivamente. Resultado análogo ao estudo em questão, no qual foi possível notar que grande parte dos entrevistados sequer tinham conhecimento do conceito de frutas, verduras e hortaliças. Depois de esclarecidos tais conceitos, alguns trabalhadores relataram que só consumiam tais alimentos quando encontrados no lixo.

Ao questionar sobre a ingestão de suco de frutas, notou-se que os participantes deste estudo apresentavam a prática do consumo de refrescos industrializados e que não detinham informações sobre as diferenças nutricionais de ambos. A carência do consumo de frutas, verduras e legumes foi mais intensa nos níveis mais graves de insegurança alimentar, fato esperado, uma vez que quanto mais intensa a situação de insegurança, maior é o comprometimento qualitativo e quantitativo da dieta. Os indivíduos que relataram consumir esses alimentos o faziam apenas uma vez por dia e nem sempre correspondiam a uma porção. 


\section{Conclusão}

A partir dos dados apresentados, evidenciou-se que a situação de insegurança alimentar moderada e grave presente entre os catadores de materiais recicláveis do município de Russas-CE é alta, refletindo o grau de marginalização social e vulnerabilidade à fome a que esses trabalhadores estão submetidos. Além disso, observou-se grande fragilidade dessa população ao desencadeamento de diversos tipos de doenças crônicas não transmissíveis, sendo esses riscos intensificados pelo agravamento da situação de insegurança alimentar.

Dentre as limitações encontradas no estudo, destaca-se a carência de pesquisas relacionadas a condição de saúde e alimentação nessa população, dificultando a comparação entre os resultados. Diante disso, recomendam-se mais estudos que possam aprofundar a compreensão das condições de vida, saúde e nutrição dos catadores de resíduos sólidos.

Tendo em vista a relação de insegurança alimentar com características socioeconômicas e o aumento do risco de DCNTs, destaca-se a necessidade de ações de responsabilidade social e políticas públicas que visem garantir a saúde e a segurança alimentar e nutricional.

Além disso, espera-se que os resultados encontrados possam despertar interesse em novas pesquisas e que auxiliem na elaboração ou na melhoria de políticas públicas locais de alimentação e nutrição que venham beneficiá-los.

\section{Colaboradores}

MRM Oliveira trabalhou em todas as etapas desde a concepção do estudo, coleta, análise e escrita do artigo; RSS Lima participou da concepção, desenho do estudo, da redação do artigo e da sua versão final; FR Silva participou da redação e revisão final do artigo; LMO Pinto participou da redação e revisão final do artigo; RMM Sampaio participou da redação e revisão final do artigo.

Conflito de interesses: Os autores declaração não haver conflito de interesses.

\section{Referências}

1. Evolução histórica do conceito de Segurança Alimentar e Nutricional (SAN) em âmbito internacional e no Brasil. In: Burity V, Frensceschini T, Valente F. Direito Humano à Alimentação Adequada no Contexto da Segurança Alimentar e Nutricional. Brasília: ABRANDH; 2010. p. 11-14.

2. Cabral CS, Lopes AG, Lopes JM, Viana RPT. Segurança Alimentar, renda e Programa Bolsa Família: estudo de coorte em munícipios do interior da Paraíba, Brasil. Cad Saúde Pública. 2014; 30(2):393-402. 
3. Custódio MB, Furquim NR, Santos GMM, Cyrillo DC. Segurança Alimentar e Nutricional e a construção de sua política: uma visão histórica. Segurança Alimentar e Nutrucional. 2001; 18(1):1-10.

4. Santos JV, Gigante DP, Domingues MR. Prevalência de insegurança alimentar em Pelotas, Rio Grande do Sul, Brasil, e estado nutricional de indivíduos que vivem nessa condição. Cad Saúde Pública. 2010; 26(1):41-49.

5. Guerra LDS, Espinosa MM, Bezerra ACD, Guimarães LV, Lima-Lopes MA. Insegurança alimentar em domić́lios com adolescentes da Amazônia Legal Brasileira: prevalência e fatores associados. Cad Saúde Pública. 2013; 29(2):335-348.

6. Custodio MB, Yuba YT, Cyrillo DC. Politica de segurança alimentar e nutricional no Brasil: uma analise da alocação de recursos. Panam Salud Pública. 2013; 33(2):144-150.

7. Holben DH, Pheley AM. Diabetes risk and obesity in food-insecure households in rural Appalachian Ohio. Prev Chronic Dis. 2006; 3(3):1-9.

8. Deus ACS, Santos AL, Gubert MB. Associação entre ocorrência e fatores de risco de doenças crônicas não transmissíveis e insegurança alimentar pregressa em adultos do Distrito Federal. Demetra: Alimentação, Nutrição \& Saúde. 2014; 9(4):893-901.

9. Mota JF, Rinaldi AEM, Pereira AF, Maestá N, Scarpin MM, Burini RC. Adaptação do índice de alimentação saudável ao guia alimentar da população brasileira. Rev Nutr. 2008; 21(5):545-552.

10. Lins APM, Sichieri R, Coutinho WF, Ramos EC, Peixoto MVM, Fonseca VM. Alimentação saudável, escolaridade e excesso de peso entre mulheres de baixa renda. Ciênc Saúde Coletiva. 2013; 2(2):357-366.

11. Oliveira CA, Rosado LEFPL, Santana AMC. Fatores predisponentes às doenças cardiovasculares: uma análise dos perfis sociais, clínicos e nutricionais de triadores de materiais recicláveis. Nutrir Gerais. 2011; 5(9):798-820.

12. Triola MF. Introdução a estatística. 7 ed. Rio de Janeiro: LCT; 1999.

13. Brasil. Ministério da Saúde. Organização Mundial da Saúde. A vigilância, o controle e a prevenção das doenças crônicas não-transmissíveis: DCNT no contexto do Sistema Único de Saúde brasileiro. Brasília: Ministério da Saúde, OPAS; 2005. 80 p.

14. Programa das Nações Unidas. Objetivos de Desenvolvimento do Milenio. Relatório Nacional de Acompanhamento. Brasília: IPEA; 2004.

15. Sousa NN, Dias MM, Sperandio N, Franceschini SCC, Priore SE. Perfil socioeconômico e insegurança alimentar e nutricional de famílias beneficiadas do Programa Bolsa Família no município de Viçosa, Estado de Minas Gerais, Brasil, em 2011: um estudo epidemiológico transversal. Epidemiol Serv Saúde. 2012; 21(4):655-662.

16. Anschau FR, Matsuo T, Segall-Corrêa AN. Insegurança alimentar entre beneficiáriarios de programas de transferência de renda. Rev Nutr. 2012; 25(2):177-189.

17. World Health Organization. Obesity: preventing and managing the gobal epidemic. Report of a WHO consultation on obesity. Geneva: WHO; 1998.

18. Mendes KL, Catão LP. Avaliação do consumo de frutas, legumes e verduras por adolescentes de Formiga - MG e sua relação com os fatores socioeconômicos. Alim Nutr. 2010; 21(2):291-296. 
19. World Health Organization. Fruit and vegetable promotion initiative: a meeting Report. Geneva: WHO; 2003.

20. Instituto Brasileiro de Geografia e Estatística. Pesquisa nacional por amostra de domicílios (PNAD) 2004: segurança alimentar. Rio de Janeiro: IBGE; 2014.139 p.

21. Instituto Brasileiro de Análises Sociais e Econômicas. Repercussões do Programa Bolsa Família na Segurança Alimentar e Nutricional das famílias beneficiadas. Rio de Janeiro: IBASE; 2008. 276 p.

22. Santos LMP, Carneiro FF, Hoefel MGL, Santos W, Nogueira TQ. The precarious livelihood in wast dumps: a report on food insecurity and hunger among recyclable waste collectors. Rev Nutr. 2013; 26(3):323-334.

23. Terrell A, Vargas A. Is food associated with chronic disease and chronic disease control? Ethnicity \& Disease. 2009; 19:S3-S6.

24. Brasil. Ministério da Saúde. Plano de ações estratégicas para o enfrentamento das doenças crônicas não transmissíveis no Brasil. Brasília: Ministério da Saúde; 2011.145 p.

25. Freitas NG, Antunes AC. Perfil nutricional de operários de uma indústria de reciclagem em Maracanaú, CE. Nutrire. 2011; 36(Supl.):270.

26. Bittencourt DC, Muttoni SMP. Perfil nutricional dos trabalhadores de cooperativas de reciclagem de resíduos sólidos da região metropolitana de Porto Alegre. Cippus-Unilassales. 2014; 3(1):149-165.

27. Velásquez-Melendez G, Schüssel MM, Brito AS, Silva AAM, Lopes-Filho JD, Kac G. Mild but not light or severe food insecurity is associated with obesity among brazilian women. J Nutr. 2011; 141(5):898-902.

28. Kamimura MA, Baxmann A, Sampaio AL, Cuppari L. Avaliação nutricional. In: Cuppari L. Nutrição clínica no adulto. São Paulo: Atheneu; 2002. p. 71-98.

Recebido: 08 de maio, 2018

Revisado: 10 de junho, 2018

Aceito: 26 de julho, 2018 
